\title{
Vitamin D receptor activation influences the ERK pathway and protects against neurological deficits and neuronal death
}

\author{
JIE YUAN $^{1 *}$, XIN GUO $^{2 *}$, ZHENGANG LIU $^{3}$, XIUQIN ZHAO $^{4}$, YAN FENG $^{5}$, \\ SIXIN SONG ${ }^{6}$, CHANGMENG CUI ${ }^{7}$ and PEI JIANG ${ }^{8}$ \\ ${ }^{1}$ Department of Neurology, Institute of Mental Health, North China University of Science and Technology; \\ Departments of ${ }^{2}$ Neurology and ${ }^{3}$ Neurosurgery, Affiliated Hospital of North China University of Science and Technology, \\ Tangshan, Hebei 063000; ${ }^{4}$ Department of Neurology, Weixian People's Hospital, Xingtai, Hebei 054700; \\ ${ }^{5}$ Department of Neurosurgery, The Second Hospital of Hebei Medical University, Shijiazhuang, Hebei 050000; \\ ${ }^{6}$ Department of Neurosurgery, Affiliated Hospital of Taishan Medical University, Taian, Shandong 271000; \\ ${ }^{7}$ Department of Neurosurgery, Affiliated Hospital of Jining Medical University, Jining, Shandong 272029; \\ ${ }^{8}$ Department of Clinical Pharmacy and Pharmacology, Jining First People's Hospital, \\ Jining Medical University, Jining, Shandong 272000, P.R. China
}

Received March 30, 2017; Accepted November 2, 2017

DOI: $10.3892 /$ ijmm.2017.3249

\begin{abstract}
Previous studies have demonstrated that global cerebral ischemia (GCI) causes neurological deficits and neuronal cell apoptosis. Calcitriol, a biologically active metabolite of vitamin $\mathrm{D}$, exerts its endocrinological influence via nuclear vitamin $\mathrm{D}$ receptor. It is being assessed as an emerging therapeutic strategy in models of various medical conditions, including acute brain injury. The purpose of the present study was to investigate the neuroprotective effects of calcitriol on GCI and further refine the potential underlying mechanisms. A total of 145 male rats were assigned to 5 groups as follows: Sham group, GCI group, calcitriol treatment group, PD98059 treatment group and vehicle-treated group. Brain water content and neurologic severity score were assessed to evaluate the brain edema and neurological deficits of rats. Histopathological changes and ultrastructures of cells were observed via hematoxylin and eosin stain and transmission electron microscopy, respectively. Immunofluorescent staining
\end{abstract}

Correspondence to: $\mathrm{Dr}$ Changmeng Cui, Department of Neurosurgery, Affiliated Hospital of Jining Medical University, 89 Guhuai Road, Jining, Shandong 272029, P.R. China

E-mail: cmcuidr1989@163.com

Dr Pei Jiang, Department of Clinical Pharmacy and Pharmacology, Jining First People's Hospital, Jining Medical University, 6 Health Road, Jining, Shandong 272000, P.R. China

E-mail: jiangpeicsu@sina.com

${ }^{*}$ Contributed equally

Key words: vitamin D, calcitriol, apoptosis, extracellular signalregulated kinase $1 / 2$, global cerebral ischemia and western blot analysis were used to assess the expression of proteins and their co-localization at the molecular level. The results demonstrated that post-GCI administration of calcitriol attenuated brain edema and improved neurological function in rats. Calcitriol also caused marked extracellular signal-regulated kinase $1 / 2$ pathway activation, and thereby attenuated neuronal apoptosis. The present study provided novel clues for understanding the mechanisms by which calcitriol exerts its neuroprotective activity in a rat model of GCI.

\section{Introduction}

Global cerebral ischemia (GCI) is a leading cause of mortality and morbidity worldwide (1). It is essential to understand the biological cascades that drive the delayed secondary phase subsequent to GCI (2). In these situations, a transient period of GCI causes selective cell death in the vulnerable cortex pyramidal cell layer days after reperfusion, which is known as delayed apoptotic neuronal death (3). However, despite extensive research, no effective treatment has been developed to repair the damage resulting from GCI in the clinic.

Vitamin D is most commonly associated with the regulation of calcium homeostasis (4). Vitamin D2 and D3 are two exogenous forms of vitamin $\mathrm{D}$, each of which is biologically inert. Their activation requires a two-step hydroxylation reaction involving 25 -hydroxylase in the liver and $1 \alpha$-hydroxylase in the kidney (5). The biologically active metabolite of vitamin D (calcitriol) exerts its endocrinological influence via a nuclear vitamin D receptor (VDR) (6). The wide distribution of VDR suggests that vitamin D may regulate various physiological pathways, such as brain development, inflammation, neurological function, cell-cycle control, immune modulation and apoptosis (7-11). In addition, vitamin D is currently being investigated as a therapeutic strategy for various neurological disease, including depressive disorder, traumatic brain injury, stroke and neurodegenerative diseases (12-15). 
The present study assessed whether post-injury treatment with calcitriol has a therapeutic effect against ischemic reperfusion-induced injury and alleviates neurological deficits. The present study was also performed to further refine the potential underlying mechanisms of calcitriol treatment. In addition, through administering PD98059 as a tool to modulate extracellular signal-regulated kinase (ERK)1/2 activation, it was assessed whether the ERK1/2 pathway has a role in neuronal apoptosis in the rat cortex.

\section{Materials and methods}

Animals. The Institutional Animal Care and Use Committee of North China University of Science and Technology (Tangshan, China) approved all experiments, which were performed according to the guidelines of the National Institutes of Health (NIH) Guide for the Care and Use of Laboratory Animals (NIH publication no. 80-23, revised 1978). All efforts were made to minimize the number of animals used and their suffering. A total of 145 male Sprague Dawley rats (age, 10-16 weeks; weight, 350-380 g) were used in the study. The rats were housed under a 12 -h light/dark cycle at $20^{\circ} \mathrm{C}$. All of the rats were fasted for 12-16 $\mathrm{h}$ prior to the experiments, but had free access to water.

Model of GCI. The transient GCI model was generated as previously described (16). In brief, ischemia was induced via bilateral carotid artery ligation under conditions of arterial hypotension. A laser Doppler monitoring system (VMS-LDF2; Moor Instruments, Axminster, UK) was used to monitor cerebral blood flow $(\mathrm{CBF})$ during the procedure. The sensor of the monitor was placed (and fixed with bone cement) 1-2 $\mathrm{mm}$ posterior and $4-5 \mathrm{~mm}$ lateral to the bregma on the left or right skull hemisphere after a small midline skin incision had been made on the same side. For blood withdrawal and re-infusion, the right jugular vein was cannulated with a silicone catheter. After heparinization (50 units), blood was quickly drawn through the jugular vein until the mean arterial pressure (MAP) attained $25-30 \mathrm{mmHg}$ and the reduction in regional $\mathrm{CBF}$ was $50 \%$ from baseline. The two common carotid arteries were then clamped with vascular clips for $10 \mathrm{~min}$, and the MAP was maintained at 25-30 $\mathrm{mmHg}$ during the ischemic period. The clips were carefully removed from both arteries and the withdrawn blood was slowly re-infused. After completion of the procedure, the rats were given $0.5 \%$ bupivacaine injections around all of the incision sites and were allowed to recover from anesthesia at room temperature.

Groups and drug administration. Experiment 1 was performed to determine the neuroprotection effects of calcitriol after GCI. A total of 135 rats were randomly assigned to 3 groups as follows: Sham group $(n=45)$, GCI group $(n=45)$ and GCI + calcitriol group $(n=45)$. In the calcitriol group, rats were intraperitoneally injected with calcitriol (Sigma-Aldrich; Merck KGaA, Darmstadt, Germany) dissolved in 5\% dimethyl sulfoxide (DMSO) and $0.9 \%$ normal saline at a dose of $1 \mu \mathrm{g} / \mathrm{kg}$ at $30 \mathrm{~min}, 12$ and $24 \mathrm{~h}$ after the GCI insult. The Sham and GCI groups received equal volumes of $5 \%$ DMSO by intraperitoneal injection at the same time. All investigations were blind and group identities were revealed only at the end of the behavioral and histological analyses.
Experiment 2 was performed to determine whether the ERK1/2 pathway was involved in neuronal death in the rat cortex. A total of 10 rats from the GCI + calcitriol group were randomly divided into two groups: The vehicle group, which received an equal volume of $20 \mu 15 \%$ DMSO $(\mathrm{n}=5$; vehicle group); and the PD98059 group, which received $5 \mu \mathrm{g}$ p-ERK1/2 inhibitor PD98059 (Bio-Rad Laboratories, Inc., Hercules, CA, USA) dissolved in $20 \mu \mathrm{l}$ 5\% DMSO (n=5; PD98059 group). Drug injection was performed at $30 \mathrm{~min}$ prior to the $\mathrm{GCI}$ insult.

Infarct measurement by 2,3,5-triphenyltetrazolium chloride (TTC) staining. At 7 days after GCI, rats were anesthetized by intraperitoneal injection with $300 \mathrm{mg} / \mathrm{kg}$ of a $10 \%$ chloral hydrate solution (Beijing Sino fir Biological Engineering Co., Ltd., Beijing, China), and intracardially perfused with isotonic sodium chloride solution. Following reperfusion, rat brains ( $n=5$ from the Sham, GCI and calcitriol groups) were removed and frozen at $-80^{\circ} \mathrm{C}$ for $5 \mathrm{~min}$. Coronal slices $(2 \mathrm{~mm})$ were stained for $20 \mathrm{~min}$ at $37^{\circ} \mathrm{C}$ with $2 \%$ TTC (Sigma-Aldrich; Merck KGaA) and were then fixed with $4 \%$ formaldehyde. The sections were scanned and the infarct area in each section was calculated by using ImageJ analysis software (version 1.46; NIH, Bethesda, MD, USA).

Hematoxylin and eosin (H\&E) stain. At 3 days after GCI, rats were anesthetized and perfused as described above, followed by perfusion with $4 \%(\mathrm{w} / \mathrm{v})$ paraformaldehyde in $0.1 \mathrm{M}$ sodium phosphate buffer ( $\mathrm{pH}$ 7.4). The brains were removed and fixed for $48 \mathrm{~h}$ in $4 \%(\mathrm{w} / \mathrm{v})$ paraformaldehyde. After fixation, brains were embedded in paraffin and sliced into $4-\mu \mathrm{m}$ coronal sections at the level of the bregma and stained with H\&E.

Transmission electron microscopy (TEM). Cortex region neurons of 9 animals (3 each from the Sham, GCI and calcitriol group) were examined via TEM at 3 days after GCI. Following perfusion, brains were removed, and the cortex region was dissected and washed in $0.1 \mathrm{M}$ phosphate buffer. Tissue samples were immersed in $2 \%$ glutaraldehyde and $1 \%$ osmium tetroxide (Sigma-Aldrich; Merck KGaA) for $2 \mathrm{~h}$ at $4^{\circ} \mathrm{C}$, and then dehydrated in a graded ethanol series. Following displacement of ethanol with propylene oxide, the tissue was embedded in Epon (both from Sigma-Aldrich; Merck KGaA) and sectioned along the coronal plane with a diamond knife (FernAnclez-hIorln 1953; Ivan Sorvall, Inc., New York, NY, USA) at a thickness of $60 \mathrm{~nm}$. The sections were stained with lead citrate and observed using a CM-120 electron microscope (Philips, Eindhoven, The Netherlands).

Evaluation of brain edema. At 1, 3 and 5 days following GCI, rat brains were separated and weighed immediately with a chemical balance to determine the wet weight (WW). Following drying in a desiccating oven for $24 \mathrm{~h}$ at $100^{\circ} \mathrm{C}$, dry tissues were weighed again to obtain the constant dry weight (DW). The percentage of water in the tissues was calculated according to the following formula: Brain water content $(\%)=(\mathrm{WW}-\mathrm{DW}) / \mathrm{WW}) \times 100 \%$.

Evaluation of neurological deficits. The neurological severity score (NSS) (17) was determined at 1, 3, 5 and 7 days after 
surgery. The investigator who performed the test was blinded to the groups. Neurological function was graded on a scale of 0-18 (normal score, 0; maximal deficit score, 18). In the severity score of injury, a score of 0 was awarded for the ability to perform the test, and the higher the score, the more severe the injury.

Terminal deoxynucleotidyl transferase deoxyuridine triphosphate nick end-labelling (TUNEL) assay. TUNEL staining was performed using an Apoptag Peroxidase in situ Apoptosis Detection kit S7100 (EMD Millipore, Billerica, MA, USA) according to the manufacturer's protocol. The nuclei of TUNEL-positive neurons that contained apoptotic bodies stained blue and were identified as apoptotic. The apoptotic cells were counted under high-power magnification (x400) and the percentage of TUNEL-positive cells among the total cells was determined.

Western blot analysis. Western blotting was performed at 3 days post-GCI. Rat cortex tissues were lysed in Tissue Protein Lysis Solution (Thermo Fisher Scientific, Inc., Waltham, MA, USA) supplemented with $5 \%$ proteinase inhibitor cocktail (Sigma-Aldrich; Merck KGaA), incubated on ice for $30 \mathrm{~min}$ and centrifuged at $15,000 \mathrm{x} \mathrm{g}$ at $22-24^{\circ} \mathrm{C}$ for $15 \mathrm{~min}$. Protein concentrations were determined using the bicinchoninic acid protein assay (reagents obtained from Nanjing Jiancheng Bioengineering Institute, Nanjing, China). Protein samples $(50 \mu \mathrm{g} /$ lane) were subjected to $10 \%$ sodium dodecyl sulfate-polyacrylamide gel electrophoresis (SDS-PAGE) and transferred to polyvinylidene difluoride membranes (Roche Diagnostics GmbH, Mannheim, Germany) for $60 \mathrm{~min}$. Nonspecific binding sites were blocked with $5 \%$ bovine serum albumin (Bio-Rad Laboratories, Inc., Hercules, CA, USA) at $22-24^{\circ} \mathrm{C}$ for $1 \mathrm{~h}$, and then incubated with various antibodies: Rabbit anti-rat VDR (cat. no. sc9164), caspase-3 (cat. no. sc7148), B-cell lymphoma-2 (Bcl-2; cat. no. sc783), ERK (cat. no. sc292838), phosphorylated (p)-ERK (cat. no. sc101760) and GAPDH (cat. no. sc25778) polyclonal antibodies (dilution, 1:1,000; Santa Cruz Biotechnology, Inc., Dallas, TX, USA) overnight at $4^{\circ} \mathrm{C}$. The membranes were then incubated with secondary antibodies (cat. no. sc2955; dilution, 1:5,000; Santa Cruz Biotechnology, Inc.) for $2 \mathrm{~h}$ at $4^{\circ} \mathrm{C}$. The immunoreactive bands were visualized using an enhanced chemiluminescence reagent (Bio-Rad Laboratories, Inc.). Blots were scanned and for densitometrical analysis, and the integrated density of pixels was quantified using Image Quant software (version 5.2; Molecular Dynamics; GE Healthcare Bio-Sciences, Pittsburgh, PA, USA).

Immunofluorescent staining. Immunofluorescent staining was performed at 3 days post-GCI as described previously. After perfusion, brains were removed, post-fixed in the same fixative for 1 day at room temperature and subsequently soaked in 30\% sucrose for 2-3 days. Subsequently, the tissues were embedded in optimal cutting temperature compound. Frozen cross sections $(12 \mu \mathrm{m})$ were prepared and examined. Following three washes with phosphate-buffered saline (PBS), for $10 \mathrm{~min}$ each, the sections were incubated with $5 \%$ donkey serum (Institute of Hematology, Chinese Academy of Medical Sciences, Tianjin, China) and $0.4 \%$
Triton X-100 (Amresco, LLC, Solon, OH, USA) at room temperature for $2 \mathrm{~h}$. The sections were incubated with primary goat polyclonal antibody to p-ERK1/2 (cat. no. sc7976; 1:200 dilution; Santa Cruz Biotechnology, Inc.), rabbit polyclonal primary antibody to caspase-3 (cat. no. sc7148; 1:200 dilution) and mouse polyclonal primary antibody to neuronal nuclei (NeuN; cat. no. sc71667; 1:200 dilution) (both from Santa Cruz Biotechnology, Inc.), respectively, overnight at $4^{\circ} \mathrm{C}$, followed by incubation with a mixture of secondary antibodies (cat. no. A-11055, A-11037 and A-32728; 1:200 dilution; Invitrogen; Thermo Fisher Scientific, Inc.) at room temperature for $2 \mathrm{~h}$. After washing with PBS 3 times for $10 \mathrm{~min}$ each, the images were captured under a fluorescence microscope (Olympus FluoView ${ }^{\mathrm{TM}}$ FV1000; Olympus, Tokyo, Japan).

Statistical analysis. SPSS 16.0 software (SPSS, Inc., Chicago, IL, USA) was used for statistical analysis. Statistical evaluation of the data was performed using an independent Student's t-test for comparison between two groups, and one-way analysis of variance for comparison among three groups, followed by post-hoc comparisons using the least significant differences or Kruskal-Wallis method. Data from the mechanical allodynia and thermal hyperalgesia tests were analysed via using repeated-measures tests. All experimental data are expressed as the mean \pm standard error and $\mathrm{P}<0.05$ was considered to indicates a statistically significant difference.

\section{Results}

Mortality of experimental rats. The mortality rate was low in rats following GCI. Two rats died during the experiments, one of which was in the GCI model group and the other was in the calcitriol treatment group. No signs of unacceptable pain/stress/illness were observed in the two rats prior to mortality.

Calcitriol decreases the brain infarct volume after GCI. As presented in Fig. 1, TTC staining revealed that the infarct ratio was significantly increased after GCI compared with that in the Sham-operated group $(\mathrm{P}<0.001)$, and intraperitoneal calcitriol injection markedly decreased the infarct ratio $(\mathrm{P}<0.005)$. This strongly indicated that vitamin $\mathrm{D}$ protects rats from hypoxiaischemia-induced brain cortex injuries.

Morphological analysis of cortical regions of the brain. At 3 days after GCI, the cortical regions of the brains were collected and morphological detection was performed via H\&E staining and TEM. As presented in Fig. 2A, the nuclei of normal neurons were round and stained pale, whereas nuclei of dying neurons were pyknotic and darkly stained after GCI. The pathological changes of cortex neurons in the calcitriol-treated group were attenuated compared with those in the GCI group. The ultrastructures of the neurons in the cortex are depicted in Fig. 2B. In the Sham group, the neurons in the cortex had large round nuclei, the double nuclear membranes were clear and complete and the outer membrane enclosed the periphery of the mitochondria. In the GCI model group, neurons were irregular and exhibited chromatin condensation, cytoplasm dissolution and vacuole formation. The nuclear membranes and cell organelles were dissolved or absent. In the calcitriol treatment group, the damage to the neurons was alleviated. 

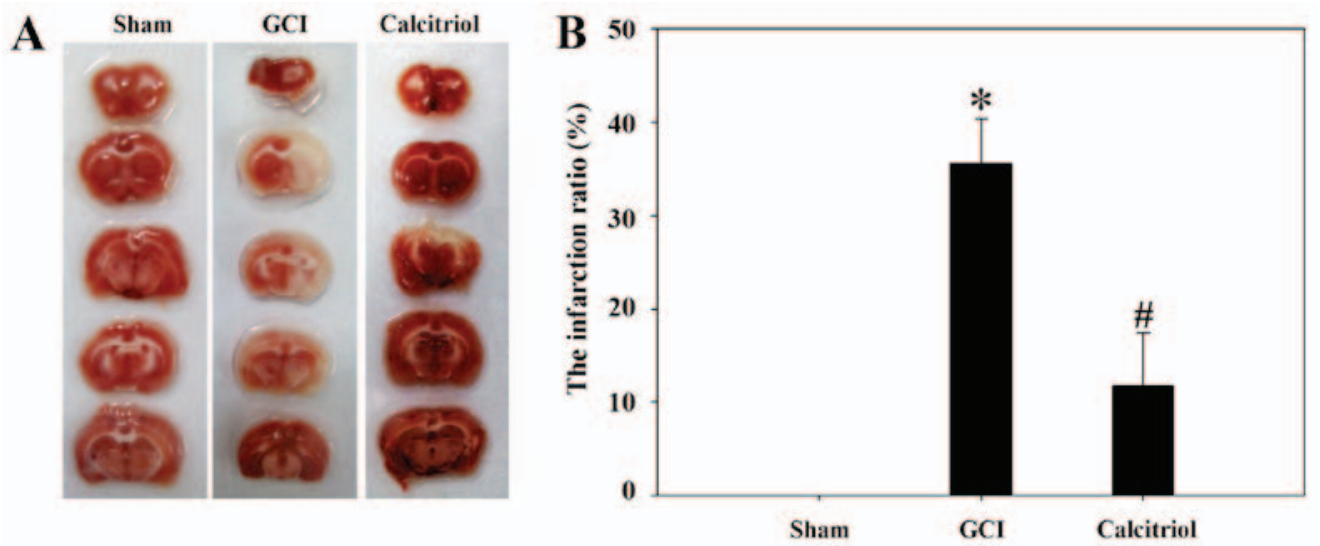

Figure 1. The effects of calcitriol on the brain infarct volume after GCI. (A) Representative images of 2,3,5-triphenyltetrazolium chloride-stained coronal sections were obtained at 7 days after GCI insult. A notable cerebral infarction region was observed in the GCI group. (B) Quantified relative region of cerebral infarction. Values are expressed as the mean \pm standard error ( $\mathrm{n}=5$ for each group). After GCI, the infarct volume was significantly increased and the infarct ratio was obviously lower after administration of calcitriol. ${ }^{\text {}} \mathrm{P}<0.001$ vs. Sham group; ${ }^{~} \mathrm{P}<0.005$ vs. GCI group. GCI, global cerebral ischemia.
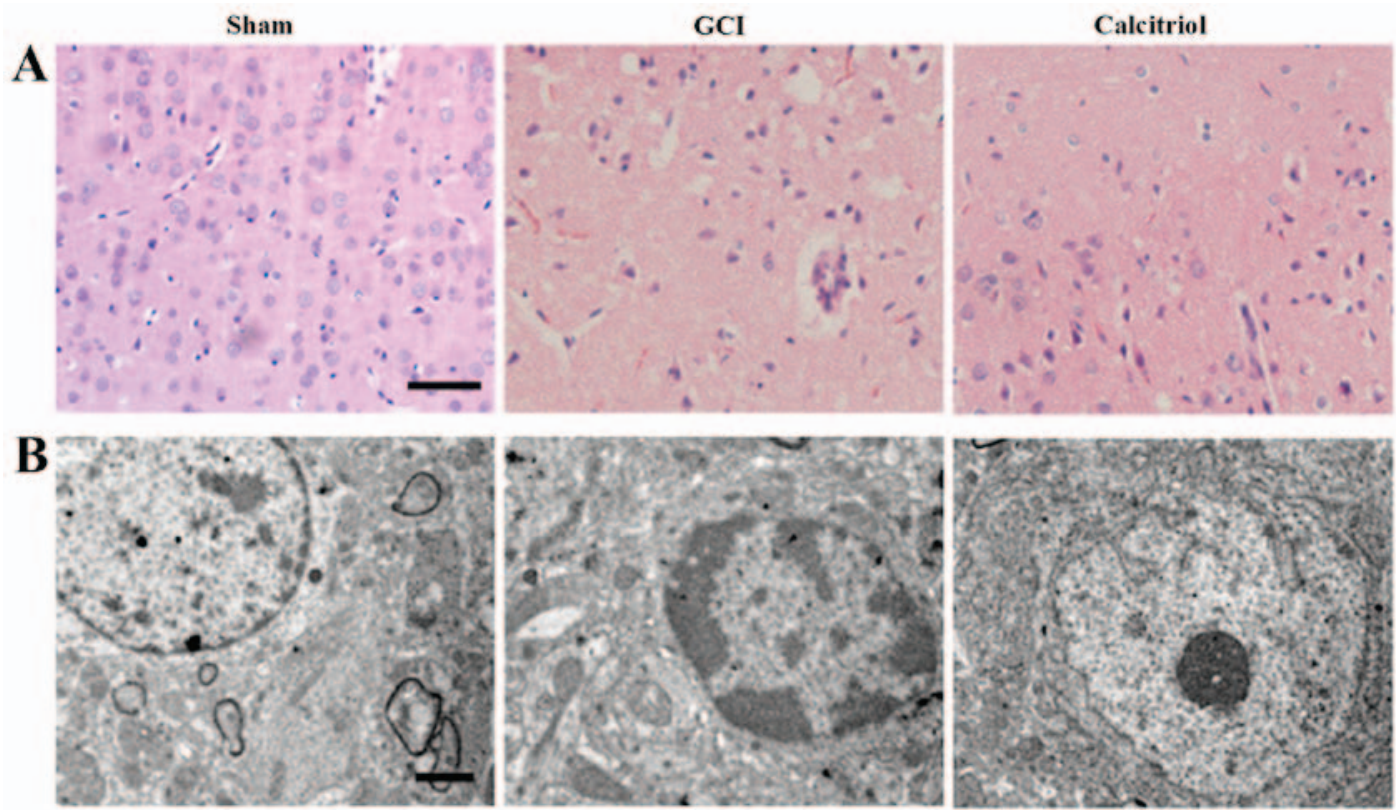

Figure 2. Morphological detection results in the cortex region of the brain after GCI. (A) Assessment of histopathologic changes in the experimental groups (hematoxylin and eosin staining; scale bar, $50 \mu \mathrm{m}$ ). The nuclei of normal neurons were round and stained pale, whereas nuclei of dying neurons were pyknotic and darkly stained after GCI. The pathological changes of cortex neurons in the calcitriol-treated group were improved compared with those in the GCI group. (B) Ultrastructures of neurons were observed via transmission electron microscopy in the experimental groups (scale bar, $2 \mu \mathrm{m}$ ). In the Sham group, the neurons in the cortex had large round nuclei, the double nuclear membranes were clear and complete, and the outer membrane enclosed the periphery of the mitochondria. In the GCI group, neurons were irregular and exhibited chromatin condensation, cytoplasm dissolution and vacuole formation. The nuclear membranes and cell organelles were dissolved or absent. In the calcitriol group, the damage to the neurons was alleviated. GCI, global cerebral ischemia.

Calcitriol attenuates GCI-induced cerebral edema. To evaluate the effects of calcitriol on brain edema, the wet-dry weight method was employed at 1, 3 and 5 days post-GCI. As presented in Fig. 3, the cerebral water content was significantly increased at 1,3 and 5 days after GCI $(\mathrm{P}<0.001$ vs. Sham group). However, treatment with calcitriol attenuated the cerebral water content at 3 and 5 days compared with that in the GCI model group $(\mathrm{P}<0.001$ and $\mathrm{P}=0.001$, respectively).

Calcitriol attenuates GCI-induced neurological deficits. Neurological deficits after GCI were expressed as the NSS in the present study. Changes in neurological deficits of rats at 1,3,5 and 7 days are depicted in Fig. 4. The rats exhibited significant neurological deficits after GCI $(\mathrm{P}<0.001)$. Post-injury administration of calcitriol significantly improved the NSS at 3, 5 and 7 days after GCI $(\mathrm{P}=0.004, \mathrm{P}=0.002$ and $\mathrm{P}=0.001$, respectively).

Increase of VDR expression after treatment of calcitriol. To further investigate the underlying mechanisms of the effect of calcitriol, the expression levels of VDR were analyzed in the cortex tissue at 3 days following the GCI insult. As demonstrated in Fig. 5, GCI caused an upregulation of VDR protein expression compared with that in Sham-operated rats $(\mathrm{P}<0.05)$, and supplementation with calcitriol further increased VDR protein expression after $\mathrm{GCI}(\mathrm{P}<0.05)$. 

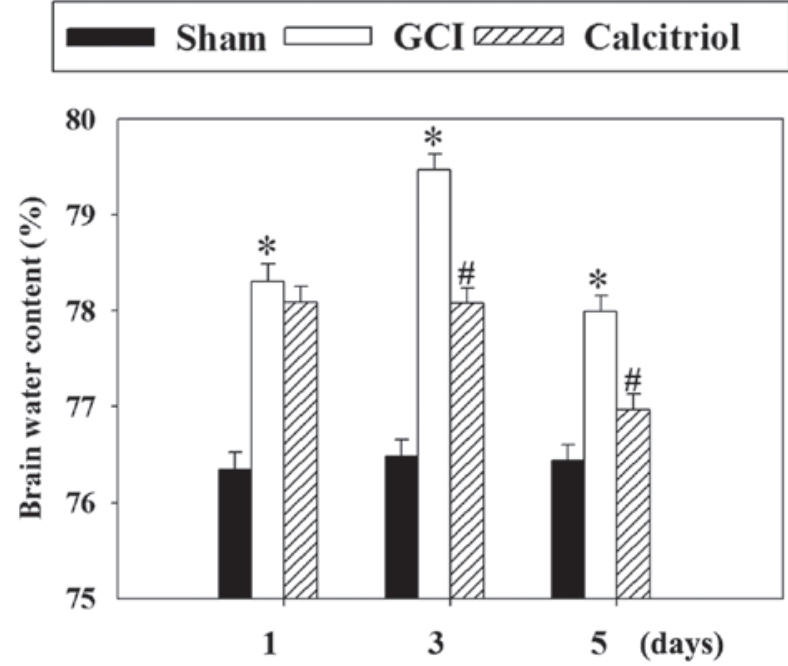

Figure 3. Effect of calcitriol treatment on brain edema. The brain water content was determined at 1,3 and 5 days following GCI. Values are expressed as the mean \pm standard error ( $\mathrm{n}=5$ for each time-point). The cerebral water content was significantly increased at 1,3 and 5 days after GCI, while it was attenuated by treatment with calcitriol at 3 and 5 days. ${ }^{*} \mathrm{P}<0.001$ vs. Sham group; " $\mathrm{P}<0.001$ vs. GCI group. GCI, global cerebral ischemia; d, days.

Calcitriol attenuates apoptotic death of cortical neurons. To assess the effect of calcitriol on neuronal apoptosis after GCI, the cortical region was subjected to a TUNEL assay at 3 days following GCI insult (Fig. 6). The results revealed few apoptotic cells in the Sham group. In the GCI model group, the nerve cells were irregularly arranged and the cell bodies were shrunken. The number of TUNEL-positive cells was significantly increased after GCI compared with that in the Sham group $(\mathrm{P}<0.001)$. However, calcitriol treatment significantly reduced the number of TUNEL-positive cells compared with that in the GCI group $(\mathrm{P}<0.001)$.

Calcitriol treatment inhibits GCI-induced changes of caspase-3 and Bcl-2 protein expression. Western blot analysis was performed to detect the expression of cleaved caspase-3 and Bcl-2 (markers of apoptosis) in the cortex region of rats at 3 days. As presented in Fig. 7, GCI caused an increase in cleaved caspase-3 expression, while attenuating $\mathrm{Bcl}-2$ expressing compared with that in the Sham-operated rats $(\mathrm{P}<0.001)$. However, compared with those in the GCI group, the levels of cleaved caspase-3 were significantly decreased in the calcitriol treatment group $(\mathrm{P}=0.006)$, while $\mathrm{Bcl}-2$ expression was markedly elevated $(\mathrm{P}=0.002)$.

Calcitriol activates the ERK1/2 pathway after GCI. Western blot analysis was performed to assess the expression of ERK1/2 and the levels of p-ERK1/2 in the Sham, GCI and calcitriol groups at 3 days. As depicted in Fig. 8, there was no significant difference in the p-ERK1/2 to ERK1/2 ratio between the GCI group and the Sham group. However, administration of calcitriol produced a significant elevation of the phosphorylation of ERK1/2 ( $\mathrm{P}=0.003$ vs. GCI group).

\section{Sham $\rightleftharpoons$ GCI एक्य Calcitriol}
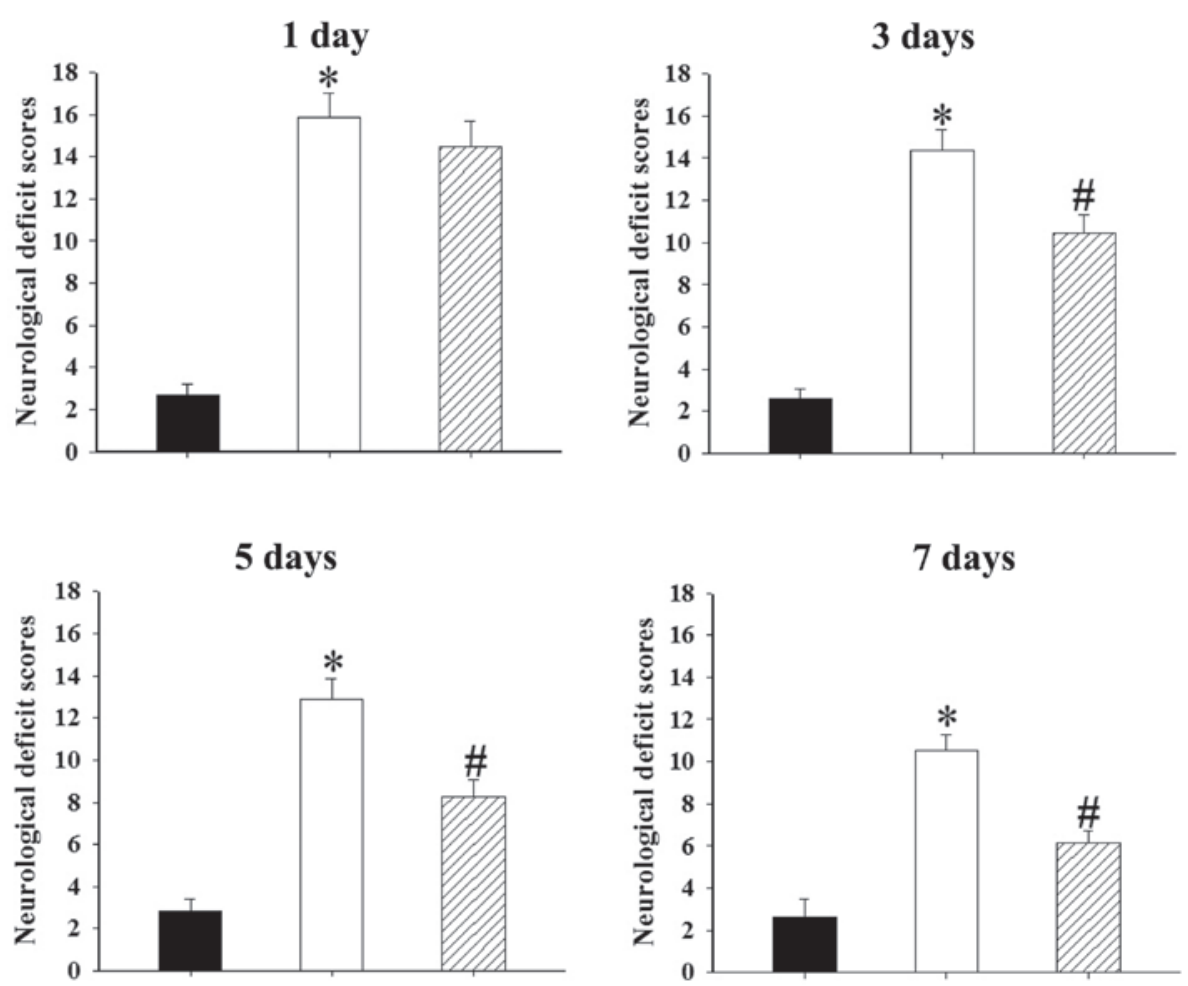

Figure 4. Effect of calcitriol treatment on GCI-induced neurological deficits. The temporal change of neurological deficits was determined at 1,3,5 and 7 days following GCI and calculated as the NSS. Values are expressed as the mean \pm standard error ( $\mathrm{n}=5$ for each group). Rats exhibited significant neurological deficits after GCI, while calcitriol significantly improved the NSS at 3, 5 and 7 days. ${ }^{*} \mathrm{P}<0.001$ vs. Sham group; ${ }^{\sharp} \mathrm{P}<0.005$ vs. GCI group. GCI, global cerebral ischemia; NSS, neurologic severity score. 
A

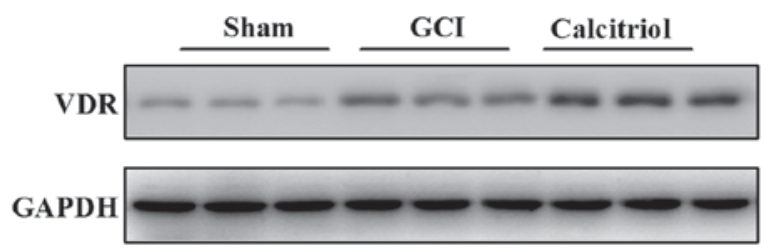

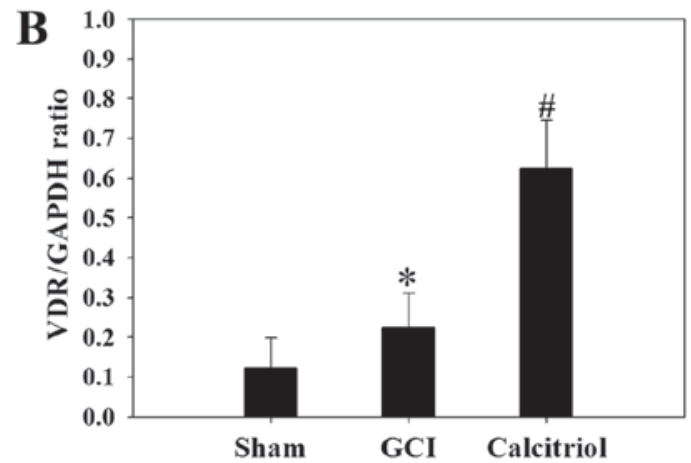

Figure 5. Effect of calcitriol treatment on VDR expression. (A) Western blot analysis was used to assess the levels of VDR in the cortex of rats at 3 days after GCI. (B) The quantitative expression levels were determined by densitometry relative to the GAPDH bands. Values are expressed as the mean \pm standard error ( $n=5$ for each group). The results indicated that the protein levels of VDR increased in the GCI group and treatment with calcitriol dramatically increased the protein expression of VDR. ${ }^{*} \mathrm{P}<0.05$ vs. Sham group; ${ }^{\text {P }}<0.05$ vs. GCI group. GCI, global cerebral ischemia; VDR, vitamin D receptor.
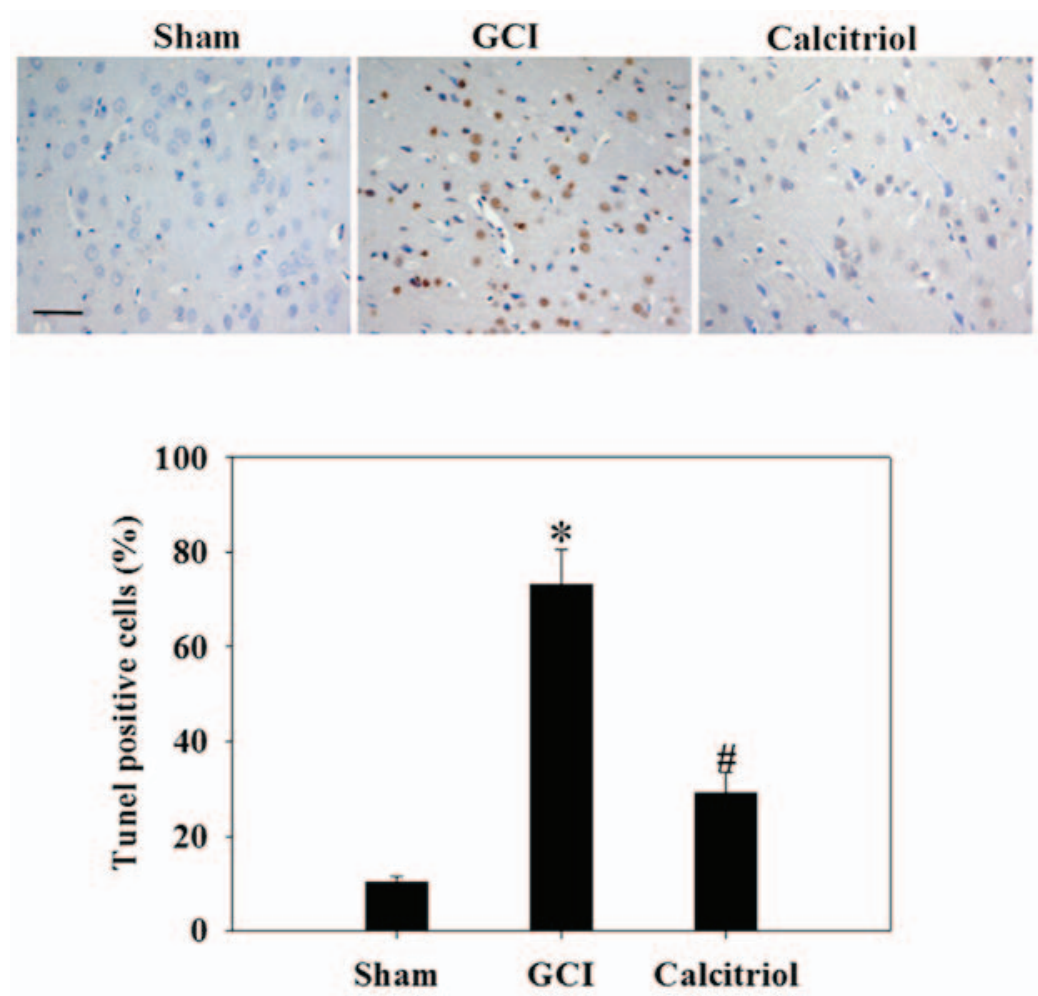

Figure 6. Effect of calcitriol on the apoptosis of neurons. The cortex region from the Sham, GCI and calcitriol groups was subjected to a TUNEL assay at 3 days after GCI (scale bar, $50 \mu \mathrm{m}$ ). Percentages of TUNEL-positive cells were determined. Values are expressed as the mean \pm standard error ( $\mathrm{n}=5$ for each group). ${ }^{*} \mathrm{P}<0.001$ vs. Sham group; ${ }^{\#} \mathrm{P}<0.001$ vs. GCI group. GCI, global cerebral ischemia; TUNEL, terminal deoxynucletidyl transferase deoxyuridine triphosphate nick end-labelling.

The ERK1/2 pathway is involved in the calcitriol-mediated inhibition of GCI-induced neuronal apoptosis. To determine whether the ERK1/2 pathway was involved in the effects of calcitriol on GCI-induced neuronal apoptosis, immunofluorescence staining was performed at 3 days after GCI. As depicted in Fig. 9, there was no fluorescence observed for caspase-3 and p-ERK1/2 in the Sham group, while staining for NeuN, a neuronal marker, was clearly visible. GCI caused a significant decrease of cells staining for NeuN, while the amount of p-ERK1/2 and caspase-3 expression was increased and co-localized in numerous cells. However, administration of calcitriol produced a marked elevation of $\mathrm{p}$-ERK1/2 and attenuation of caspase-3. The number of cells with co-localization of p-ERK $1 / 2$ and caspase- 3 was significantly decreased. These co-staining results indicated that the ERK1/2 pathway may participate in the inhibition of GCI-induced neuronal apoptosis after calcitriol treatment.

PD98059 inhibits Bcl-2 and p-ERK1/2 and increases caspase-3 after GCI in the presence of calcitriol. To further investigate whether the attenuation of apoptosis following calcitriol treatment was regulated by the ERK1/2 signaling pathway, a p-ERK1/2 inhibitor, PD98059, was used in the present study. The alterations in apoptotic cytokine levels and cell signaling following intervention with PD98059 prior to the GCI insult are depicted in Fig. 7. Of note, PD98059 inhibited 

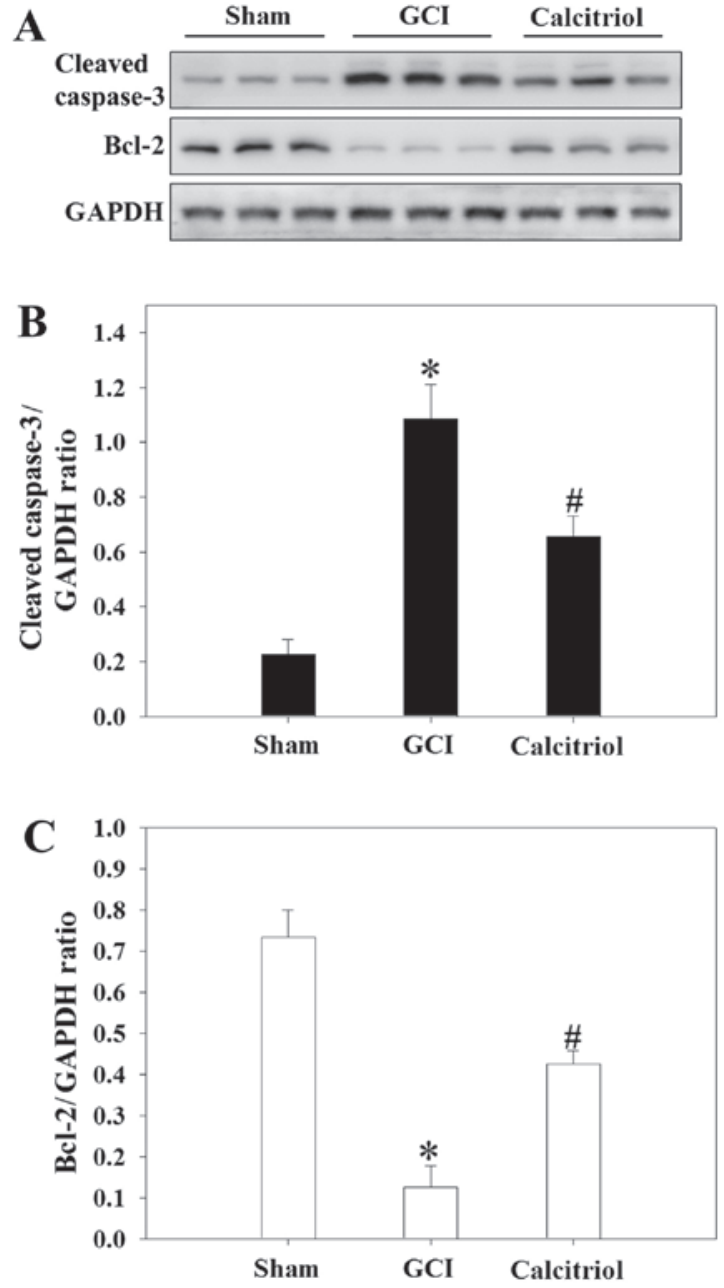

Figure 7. Effect of calcitriol on cleaved caspase-3 and Bcl-2 expression after GCI. (A) Western blot analysis indicating the levels of cleaved caspase- 3 and Bcl-2 in the cortex of rats at 3 days. (B and C) The quantitative results of cleaved caspase- 3 and $\mathrm{Bcl}-2$ were determined as the densitometric ratio vs. the GAPDH bands. Values are expressed as the mean \pm standard error $(\mathrm{n}=5$ for each group). ${ }^{*} \mathrm{P}<0.001$ vs. Sham group; ${ }^{*} \mathrm{P}=0.006$ vs. $\mathrm{GCI}$ group (cleaved caspase-3) and ${ }^{\#} \mathrm{P}=0.002$ vs. GCI group (Bcl-2). GCI, global cerebral ischemia; Bcl-2, B-cell lymphoma-2.

the expression of p-ERK1/2 in the presence of calcitriol after GCI $(\mathrm{P}<0.001)$ (Fig. 10A and B). Furthermore, the levels of caspase-3 were significantly increased, whereas Bcl-2 was significantly decreased in the PD98059 group compared with those in the calcitriol and GCI-treated vehicle group $(\mathrm{P}=0.014$ and $\mathrm{P}<0.001$, respectively) (Fig. $10 \mathrm{C}$ and $\mathrm{D}$ ).

\section{Discussion}

The purpose of the present study was to investigate the neuroprotective effects of calcitriol on GCI. H\&E staining is a macroscopic and readily available method to assess histopathological changes. Calcitriol treatment was revealed to markedly attenuate injury. In the calcitriol group, the structure of the brain tissue was better and the number of neurons was greater than that in the GCI group. The ultrastructures of neurons observed via TEM also indicated that calcitriol treatment alleviated the damage in the cortex. In addition, GCI-induced neurological deficits and brain edema were also suppressed by calcitriol treatment. At the molecular level, VDR expression in the
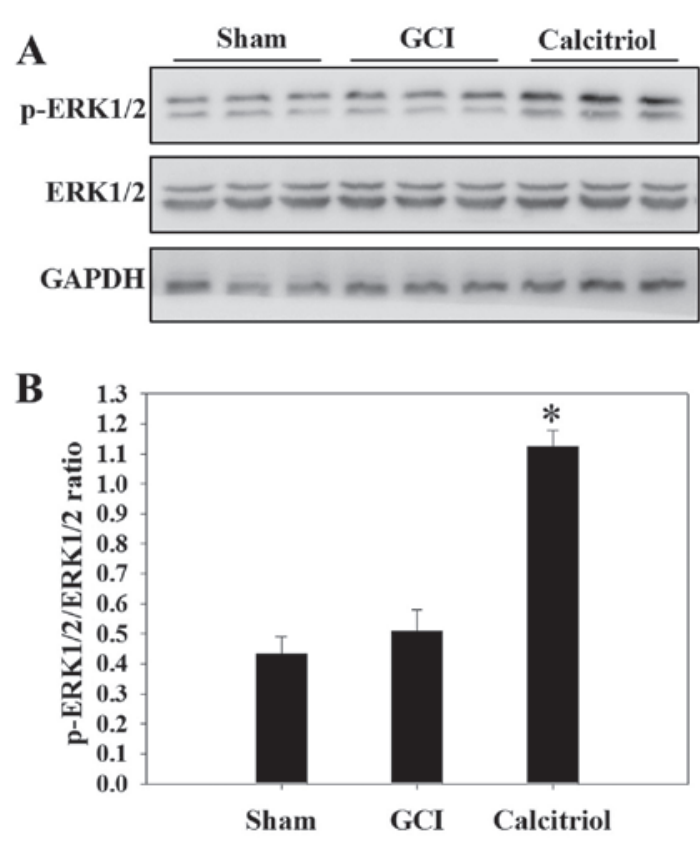

Figure 8. Effect of calcitriol on the ERK1/2 pathway. (A) Western blot analysis indicated the levels of ERK1/2 and p-ERK1/2 in the cortex of rats at 3 days after GCI. (B) The quantitative results for $\mathrm{p}$-ERK1/2 were expressed as the ratio to ERK1/2 as determined by densitometry of the western blot bands. Values are expressed as the mean \pm standard error ( $\mathrm{n}=5$ for each group). ${ }^{*} \mathrm{P}=0.003$ vs. GCI group. GCI, global cerebral ischemia; $\mathrm{p}-\mathrm{ERK}$, phosphorylated extracellular signal-regulated kinase.

cortex was significantly elevated following calcitriol treatment. Calcitriol also inhibited GCI-induced activation of caspase-3 as well as attenuation of Bcl-2. Furthermore, the effect of calcitriol was associated with activation of the ERK pathway. The results were consistent with those of previous studies reporting that calcitriol exerted neuroprotective effects in various in vitro and in vivo models (18-21). Therefore, calcitriol has the potential to become a novel therapeutic for GCI.

The primary injury occurs simultaneously with GCI, leading to a disruption of oxygen supply to the brain that contributes to immediate (necrotic) cell death (2). Thereafter, oxygen free radical-mediated lipid peroxidation and brain edema formation appear to be fundamental mechanisms of secondary damage associated with GCI (2). Sequential activation of caspases, a family of proteases, has a pivotal role in cellular apoptosis in the central nervous system (22). Apoptotic stimuli, including ischemic injury, trigger the activation of initiator caspases and subsequently the caspase cascade, finally leading to apoptotic cell death (23). Of the various subtypes of caspases, caspases- 3 is the principal caspase actively causing neuronal cell death (23). Numerous studies have demonstrated that caspase- 3 activity increases after cerebral ischemia/reperfusion injury $(24,25)$. In the present study, caspase-3 was induced by GCI. However, treatment with calcitriol significantly inhibited GCI-induced expression of cleaved caspase-3. The results of the TUNEL assay revealed few apoptotic cells in the Sham group, while a significant increase of apoptotic cells was noted in the GCI model group. However, the apoptotic rate was significantly lower in the calcitriol treatment group compared with that in the GCI group. Taken together, reduction of apoptosis is an important mechanism by which calcitriol exerts its neuroprotective effects. 


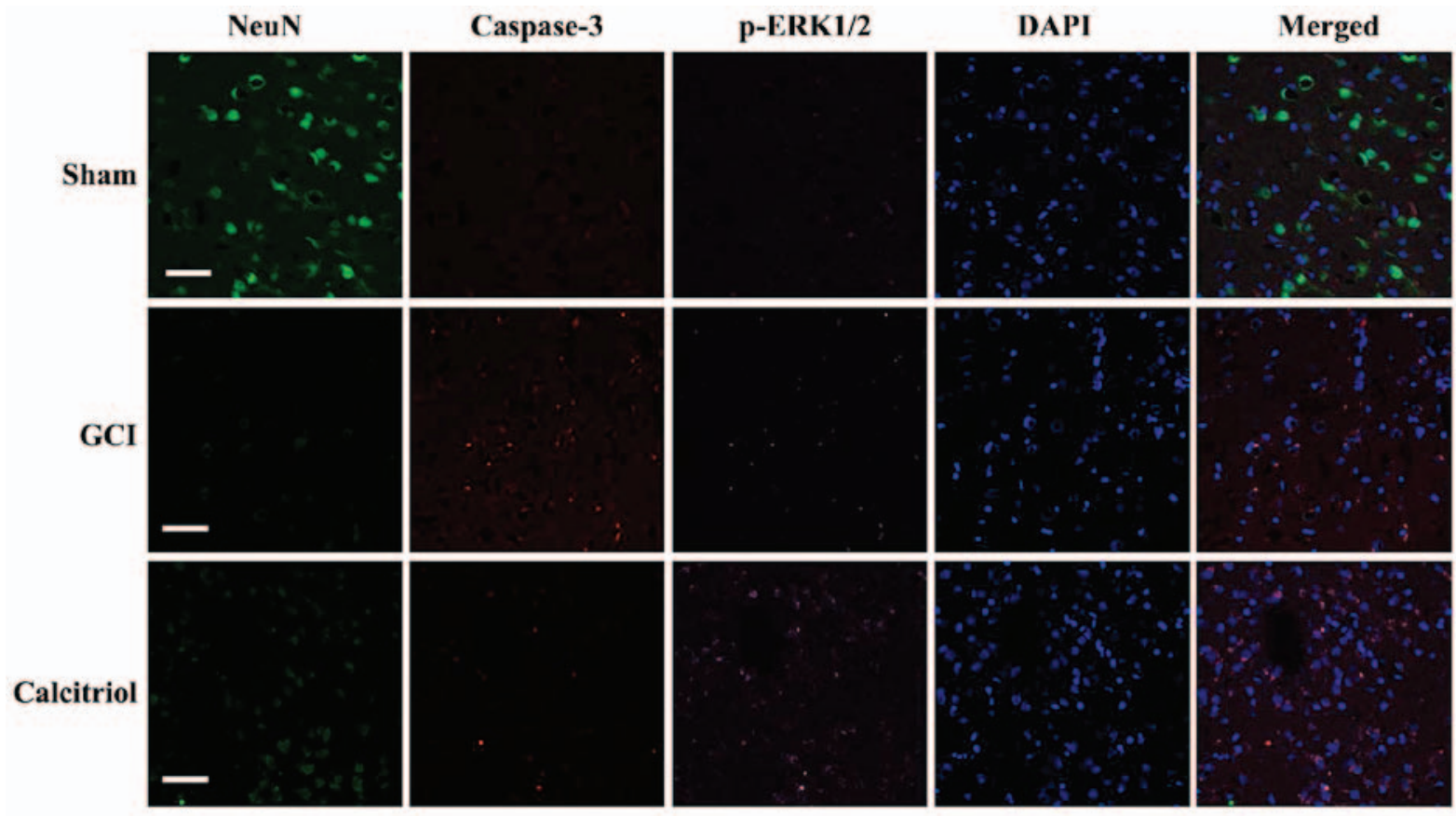

Figure 9. Fluorescence staining for NeuN, caspase-3 and p-ERK1/2 in rat cortex, and the merged images. Immunofluorescence staining was performed at 3 days in Sham, GCI and calcitriol groups ( $\mathrm{n}=5$ for each group; scale bar, $50 \mu \mathrm{m}$; green, NeuN; red, caspase-3; purple, p-ERK1/2; blue, DAPI). GCI, global cerebral ischemia; p-ERK, phosphorylated extracellular signal-regulated kinase.

A
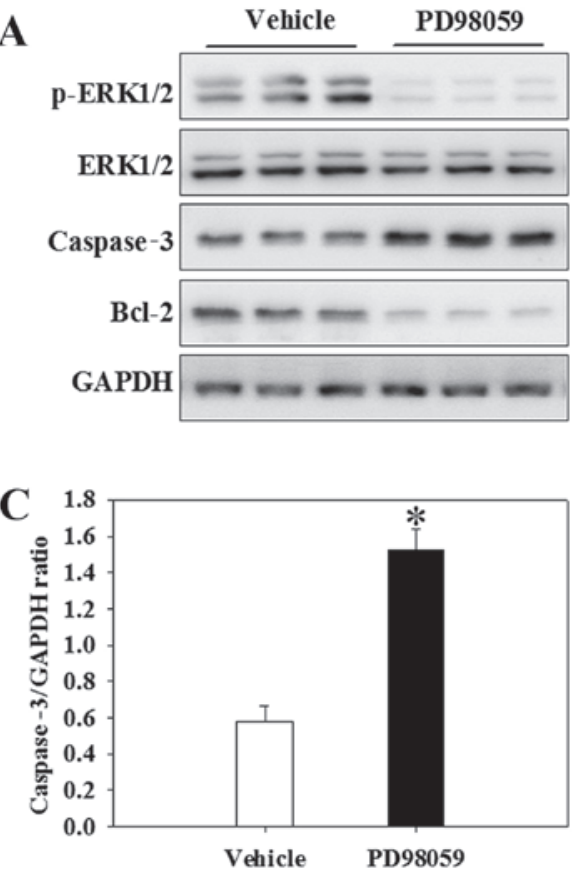

B

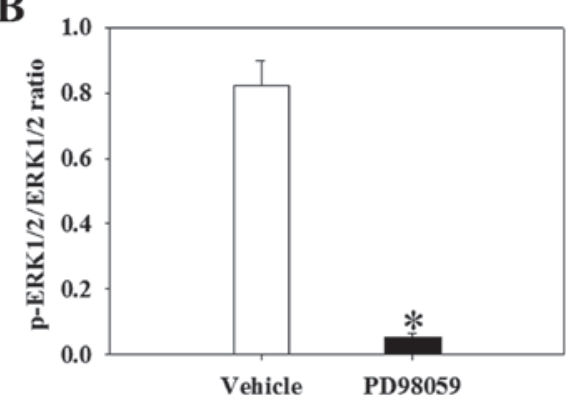

D

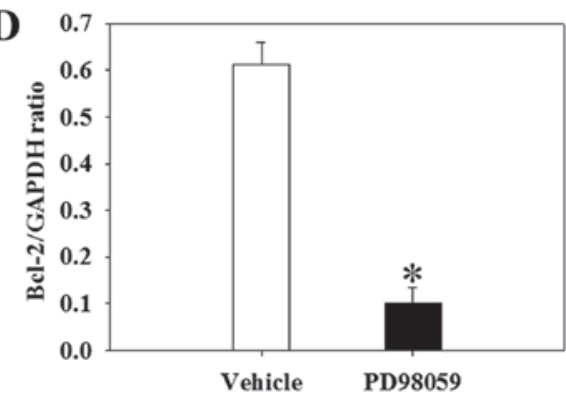

Figure 10. Effects of PD98059 on calcitriol-treated rats. (A) Western blot analysis was used to detect the expression levels of p-ERK1/2, caspase-3 and Bcl-2 in the cortex of rats at 3 days. (B) The quantitative results for p-ERK1/2 were expressed as the ratio to ERK1/2 as determined by densitometry of the western blot bands. The quantitative results of (C) caspase-3 and (D) Bcl-2 were expressed as the densitometric ratio vs. the GAPDH bands. Values are expressed as the mean \pm standard error ( $\mathrm{n}=5$ for each group). ${ }^{*} \mathrm{P}<0.001$ vs. vehicle group in ( $\mathrm{B}$ and $\left.\mathrm{D}\right)$; and ${ }^{~} \mathrm{P}=0.014$ vs. vehicle group in $(\mathrm{C})$. GCI, global cerebral ischemia; p-ERK, phosphorylated extracellular signal-regulated kinase; Bcl-2, B-cell lymphoma-2.

The intrinsic pathway of apoptosis is characterized by mitochondrial outer membrane permeabilization, death-inducing signaling complex formation, DNA fragmentation and caspase- 3 activation (26). These events have been demonstrated to be associated with ERK1/2 pathway activation $(27,28)$. In the present study, p-ERK $1 / 2$ and caspase- 3 were co-localized, which indicated that the ERK1/2 pathway may participate in the calcitriol-mediated inhibition of neuronal apoptosis induced by GCI. Furthermore, PD98059 inhibited the expression of p-ERK1/2 while significantly increasing the expression levels of caspase- 3 , whereas Bcl-2 was significantly decreased. Therefore, it is conceivable that the activation of 
the ERK1/2 pathway induced by VDR activation may have an essential role in the evasion of apoptosis after GCI.

In conclusion, the results of the present study demonstrated that post-GCI administration of calcitriol attenuate brain edema and improved neurological function in rats. Calcitriol also caused a marked activation of the ERK1/2 pathway with subsequent attenuation of neuronal apoptosis. The present study provided novel clues for understanding the mechanisms by which calcitriol exerts its neuroprotective effects in a rat model of GCI.

\section{References}

1. Zhao P, Zhou R, Zhu XY, Hao YJ, Li N, Wang J, Niu Y, Sun T, $\mathrm{Li}$ YX and Yu JQ: Matrine attenuates focal cerebral ischemic injury by improving antioxidant activity and inhibiting apoptosis in mice. Int J Mol Med 36: 633-644, 2015.

2. Han J, Sun L, Xu Y, Liang H and Cheng Y: Activation of PPAR $\gamma$ by 12/15-lipoxygenase during cerebral ischemia-reperfusion injury. Int J Mol Med 35: 195-201, 2015.

3. Bao C, Wang Y, Min H, Zhang M, Du X, Han R and Liu X Combination of ginsenoside Rg1 and bone marrow mesenchymal stem cell transplantation in the treatment of cerebral ischemia reperfusion injury in rats. Cell Physiol Biochem 37: 901-910, 2015.

4. Hellman P, Liu W, Westin G, Törmä H and Akerström G: Vitamin D and retinoids in parathyroid glands (Review). Int J Mol Med 3: 355-361, 1999.

5. Hwang JH, Wang T, Lee KS, Joo JK and Lee HG: Vitamin D binding protein plays an important role in the progression of endometriosis. Int J Mol Med 32: 1394-1400, 2013.

6. Bouillon R, Carmeliet G, Verlinden L, van Etten E, Verstuyf A, Luderer HF, Lieben L, Mathieu C and Demay M: Vitamin D and human health: Lessons from vitamin D receptor null mice. Endocr Rev 29: 726-776, 2008.

7. Eyles DW, Burne THJ and McGrath JJ: Vitamin D, effects on brain development, adult brain function and the links between low levels of vitamin D and neuropsychiatric disease. Front Neuroendocrinol 34: 47-64, 2013.

8. Pittas AG, Harris SS, Stark PC and Dawson-Hughes B: The effects of calcium and vitamin D supplementation on blood glucose and markers of inflammation in nondiabetic adults. Diabetes Care 30: 980-986, 2007.

9. Jorde R, Mathiesen EB, Rogne S, Wilsgaard T, Kjærgaard M, Grimnes $\mathrm{G}$ and Schirmer H: Vitamin D and cognitive function: The Troms $\varnothing$ Study. J Neurol Sci 355: 155-161, 2015.

10. Segaert S, Degreef H and Bouillon R: Vitamin D receptor expression is linked to cell cycle control in normal human keratinocytes. Biochem Biophys Res Commun 279: 89-94, 2000.

11. McGuire TF, Trump DL and Johnson CS: Vitamin D(3)-induced apoptosis of murine squamous cell carcinoma cells. Selective induction of caspase-dependent MEK cleavage and up-regulation of MEKK-1. J Biol Chem 276: 26365-26373, 2001.

12. Tang H, Hua F, Wang J, Sayeed I, Wang X, Chen Z, Yousuf S, Atif $F$ and Stein DG: Progesterone and vitamin D: Improvement after traumatic brain injury in middle-aged rats. Horm Behav 64 527-538, 2013.

13. Jiang P, Xue Y, Li HD, Liu YP, Cai HL, Tang MM and Zhang LH: Dysregulation of vitamin D metabolism in the brain and myocardium of rats following prolonged exposure to dexamethasone. Psychopharmacology (Berl) 231: 3445-3451, 2014.
14. Balden R, Selvamani A and Sohrabji F: Vitamin D deficiency exacerbates experimental stroke injury and dysregulates ischemia-induced inflammation in adult rats. Endocrinology 153 : 2420-2435, 2012.

15. Nissou MF, Guttin A, Zenga C, Berger F, Issartel JP and Wion D: Additional clues for a protective role of vitamin D in neurodegenerative diseases: 1,25-dihydroxyvitamin D3 triggers an anti-inflammatory response in brain pericytes. J Alzheimers Dis 42: 789-799, 2014

16. Lee H, Park YH, Jeon YT, Hwang JW, Lim YJ, Kim E, Park SY and Park HP: Sevoflurane post-conditioning increases nuclear factor erythroid 2-related factor and haemoxygenase-1 expression via protein kinase $\mathrm{C}$ pathway in a rat model of transient global cerebral ischaemia. Br J Anaesth 114: 307-318, 2015.

17. Chen J, Li Y, Wang L, Zhang Z, Lu D, Lu M and Chopp M: Therapeutic benefit of intravenous administration of bone marrow stromal cells after cerebral ischemia in rats. Stroke 32: 1005-1011, 2001

18. Kajta M, Makarewicz D, Ziemińska E, Jantas D, Domin H, Lasoń W, Kutner A and Łazarewicz JW: Neuroprotection by co-treatment and post-treating with calcitriol following the ischemic and excitotoxic insult in vivo and in vitro. Neurochem Int 55: 265-274, 2009.

19. Orme RP, Bhangal MS and Fricker RA: Calcitriol imparts neuroprotection in vitro to midbrain dopaminergic neurons by upregulating GDNF expression. PLoS One 8: e62040, 2013.

20. Fu J, Xue R, Gu J, Xiao Y, Zhong H, Pan X and Ran R: Neuroprotective effect of calcitriol on ischemic/reperfusion injury through the NR3A/CREB pathways in the rat hippocampus. Mol Med Rep 8: 1708-1714, 2013.

21. Alkharfy KM, Al-Daghri NM, Yakout SM and Ahmed M: Calcitriol attenuates weight-related systemic inflammation and ultrastructural changes in the liver in a rodent model. Basic Clin Pharmacol Toxicol 112: 42-49, 2013.

22. Talanian RV, Quinlan C, Trautz S, Hackett MC, Mankovich JA, Banach D, Ghayur T, Brady KD and Wong WW: Substrate specificities of caspase family proteases. J Biol Chem 272: 9677-9682, 1997.

23. Tamatani M, Ogawa S, Niitsu Y and Tohyama M: Involvement of Bcl-2 family and caspase-3-like protease in NO-mediated neuronal apoptosis. J Neurochem 71: 1588-1596, 1998.

24. Chiarini A, Liu D, Armato U and Dal Prà I: Bcl10 crucially nucleates the pro-apoptotic complexes comprising PDK1, PKC $\xi$ and caspase- 3 at the nuclear envelope of etoposide-treated human cervical carcinoma C4-I cells. Int J Mol Med 36: 845-856, 2015.

25. Gu YJ, Sun WY, Zhang S, Li XR and Wei W: Targeted blockade of JAK/STAT3 signaling inhibits proliferation, migration and collagen production as well as inducing the apoptosis of hepatic stellate cells. Int J Mol Med 38: 903-911, 2016.

26. Wu LF, Wei BL, Guo YT, Ye YQ, Li GP, Pu ZJ and Feng JL: Apoptosis induced by adenosine involves endoplasmic reticulum stress in EC109 cells. Int J Mol Med 30: 797-804, 2012.

27. Li Y, Lu X, Qi H, Li X, Xiao X and Gao J: Ursolic acid induces apoptosis through mitochondrial intrinsic pathway and suppression of ERK1/2 MAPK in HeLa cells. J Pharmacol Sci 125: 202-210, 2014

28. Yang H, Xiong J, Luo W, Yang J and Xi T: 8-Methoxypsoralen induces intrinsic apoptosis in HepG2 cells: Involvement of reactive oxygen species generation and ERK1/2 pathway inhibition. Cell Physiol Biochem 37: 361-374, 2015.

This work is licensed under a Creative Commons Attribution-NonCommercial-NoDerivatives 4.0 International (CC BY-NC-ND 4.0) License. 